\section{Projeto Bambuí: um estudo de base populacional da prevalência e dos fatores associados à necessidade de cuidador entre idosos}

\author{
The Bambuí Health and Aging Study (BHAS): \\ a population-based cohort study of prevalence \\ and factors associated with the needs \\ of caregivers for the elderly
}

Karla C. Giacomin 1,2

Elizabeth Uchôa 2

Josélia O. A. Firmo 2

Maria Fernanda Lima-Costa 2

\author{
1 Programa de \\ Pós-graduação em Saúde \\ Pública, Universidade \\ Federal de Minas Gerais, \\ Belo Horizonte, Brasil. \\ 2 Núcleo de Estudos \\ em Saúde Pública \\ e Envelhecimento \\ Centro de Pesquisas René \\ Rachou, Fundação Oswaldo \\ Cruz/Faculdade de Medicina, \\ Universidade Federal \\ de Minas Gerais, \\ Belo Horizonte, Brasil. \\ Correspondência \\ K. C. Giacomin \\ Núcleo de Estudos em Saúde \\ Pública e Envelhecimento, \\ Centro de Pesquisas René \\ Rachou, Fundação Oswaldo \\ Cruz/Faculdade de Medicina, \\ Universidade Federal \\ de Minas Gerais. \\ Av. Augusto de Lima 1715, \\ Belo Horizonte, $M G$ \\ 30190-002, Brasil. \\ giacomin@cpqrr.fiocruz.br
}

\begin{abstract}
The objective of this study was to determine the prevalence and factors associated with the needs of caregivers for older adults living in the community. All residents ( $n=1,742)$ of Bambuí, Minas Gerais State, Brazil (15,000 inhabitants) aged $\geq 60$ years were selected. Of these, $92 \%$ were interviewed and $86 \%$ were examined. The dependent variable "need for a caregiver" was defined as the inability to perform at least one of the basic activities of daily living and/or a Mini Mental score under 13. Some 23\% of the elderly required caregivers. After adjustment for confounding, independent and positive associations with the need for a caregiver were found for: age, single marital status, history of alcohol abuse, hypertension, obesity, and use of $\geq 2$ prescription drugs. Independent and negative associations were found for: level of schooling, familiar income, living alone, total cholesterol > $240 \mathrm{mmHg}$, and having a private health plan. Those requiring caregivers presented evidence of worse socioeconomic and health status. The study provides evidence that care of the dependent elderly is a public health problem.
\end{abstract}

Frail Elderly; Caregivers; Aging Health; Health Conditions

\section{Introdução}

O envelhecimento populacional acarreta profundas implicações sobre as políticas sociais e representa um dos maiores desafios da saúde pública contemporânea 1,2. As doenças dos idosos, em geral, são crônicas e múltiplas, perduram por vários anos, demandam mais serviços de saúde e exigem cuidados permanentes 1 . Como lembra Caldas 3 , doenças podem gerar incapacidades e causar dependência, com ônus crescentes sobre o idoso, a família e o sistema de saúde. O Brasil vem envelhecendo de forma rápida e intensa e já conta hoje com mais de 14,5 milhões de idosos 4 . Apesar disso, até hoje no Brasil, pouco se conhece do impacto, sobre o sistema de saúde, de idosos que estão dependentes e necessitam de um cuidador.

É importante compreender o quadro teórico proposto pela Organização Mundial da Saúde (OMS) 5 para a classificação das deficiências, incapacidades e dependências. A incapacidade representa as conseqüências da deficiência de um órgão ou sistema sobre o funcionamento do indivíduo em termos de limitações de função ou de restrição de atividades 6,7. Chamam-se limitações funcionais as restrições em realizar ações físicas e mentais fundamentais para a vida diária do indivíduo, em comparação às pessoas de mesmo sexo e faixa etária $6,7,8$. A dependência seria a distância entre a incapacidade que o indivíduo apresenta e os re- 
cursos materiais e sociais de que ele dispõe para superá-la 6 . Essa distância lhe confere uma desvantagem social 6 . Porém, o processo que decorre entre o aparecimento de uma deficiência orgânica e o surgimento da dependência não é unidirecional, nem linear e envolve efeitos secundários onde uma incapacidade funcional primária pode levar a futuras patologias, limitações e a outras incapacidades 9,10. Assim, Verbrugge \& Jette 7 definem a incapacidade funcional como sendo a dificuldade experimentada em realizar atividades em qualquer domínio da vida (autocuidado, cuidado com a casa e trabalho) devido a um problema físico ou de saúde. Ela pode ter origem em pelo menos um componente, que pode coexistir ou não com outros(s) - físico e/ou cognitivo e/ou social. Diversos estudos discutem separadamente os fatores associados aos déficits físicos 8,11 e cognitivos 11,12. No entanto, são necessários estudos que considerem a imbricação entre os componentes da incapacidade.

Em caso de incapacidade, a dependência é a necessidade de o indivíduo recorrer à ajuda humana e/ou técnica para realizar as atividades da vida diária. A dependência também é um processo dinâmico, que pode ser modificado ou prevenido se houver ambiente e assistência adequados 3 . Muitos estudos usam a dependência (ter a ajuda de alguém para realizar uma atividade) como um indicador de incapacidade. Verbrugge \& Jette 7 propõem um modelo do processo de tornar-se incapaz, em que categorizam os fatores de risco para incapacidade em: (1) predisponentes (fatores de risco existentes antes do processo de incapacidade ser instalado); (2) intra-individuais (estilo de vida, atributos psicossociais, mudanças de comportamento, maneiras de lidar com as dificuldades e com as modificações de atividades que podem afetar o processo de incapacidade); e (3) extra-individuais (suporte social, intervenções de serviços de saúde e de reabilitação, uso de medicamentos, suportes externos e o ambiente físico e social).

Néri \& Sommerhalder 13 ressaltam que a existência de uma incapacidade funcional é o que determina a necessidade de um cuidador. Nos países desenvolvidos, o cuidador já é assimilado como um parceiro da equipe de saúde, mas ainda não tem seu papel reconhecido no sistema de saúde brasileiro. Na Inglaterra, estima-se que mais de 6 milhões de pessoas sejam cuidadores de indivíduos dependentes, em sua maioria idosos 14. Pelo nosso conhecimento, não existem estudos no Brasil explorando esse fato. Tal lacuna é importante porque a necessidade de cuidador é um fator a mais a ser considerado no planejamento de políticas públicas de saúde para idosos.

O presente trabalho, desenvolvido como parte do Projeto Bambuí, tem por objetivo determinar a prevalência de idosos que necessitam de cuidador na comunidade, bem como indicar os fatores associados a essa necessidade, propostos por Verbrugge \& Jette 7 (fatores de risco predisponentes, intra e extra-individuais).

\section{Metodologia}

\section{Área estudada}

O Projeto Bambuí é um estudo de coorte de base populacional desenvolvido na sede do $\mathrm{Mu}$ nicípio de Bambuí, oeste de Minas Gerais, Brasil, que possuía, em 1996, 21.187 habitantes, 70\% dos quais vivendo na sede 15 . Nessa época, a expectativa de vida ao nascer era de 70,2 anos e $75 \%$ das mortes ocorriam em pessoas de cinqüenta anos ou mais. As principais causas de morte entre os habitantes do Município de Bambuí naquele ano foram doença cerebrovascular, doença de Chagas e infarto agudo do miocárdio (taxas de mortalidade $=113,3 ; 61,4$ e 42,5 por 100 mil habitantes, respectivamente) 16 . A alta taxa de mortalidade por doença de Chagas entre os residentes da comunidade é conseqüência de infecção no passado por Trypanosoma cruzi. Embora a transmissão da referida infecção já tenha sido interrompida há pelo menos vinte anos, as taxas de soropositividade para $T$. cruzi entre idosos permanecem elevadas devido ao efeito de coorte 17.

Não há instituição de longa permanência para idosos em Bambuí.

\section{População estudada}

Os participantes da coorte foram identificados por meio de um censo completo realizado na cidade pela equipe do projeto. Todos os moradores com sessenta anos ou mais de idade em 01 de janeiro de 1997 ( $n=1.742$ ) foram selecionados para participar da linha de base do estudo, constituída em 1997. Foram entrevistados 1.606 idosos (92\%), dos quais 1.495 (86\%) foram examinados (exames laboratoriais, medidas físicas e eletrocardiograma). Os participantes da coorte eram semelhantes à população da 
cidade em relação a todas as características sociodemográficas investigadas. Maiores detalhes podem ser encontrados em Lima-Costa et al. 16. Para o presente trabalho todos os participantes da linha de base da coorte de Bambuí foram selecionados.

\section{Variáveis do estudo}

\section{- Variável dependente}

A variável dependente deste trabalho é a necessidade de cuidador, definida por: (1) relato de incapacidade (não conseguir) para realizar pelo menos uma das seguintes atividades da vida diária: comer, vestir-se, ir ao banheiro, banhar-se, levantar-se de uma cadeira sem apoio 18; e/ou (2) verificação de incapacidade cognitiva determinada por um escore inferior a 1319 no Mini Mental State Examen 20. Esse ponto de corte foi adotado devido à alta proporção de idosos com menos de oito anos completos de escolaridade na comunidade estudada.

\section{- Variáveis independentes}

Foram considerados quatro conjuntos de variáveis independentes: (a) características sóciodemográficas predisponentes: sexo, idade, estado conjugal, anos completos de escolaridade, renda domiciliar mensal em salários-mínimos da época, procedência e tipo de arranjo domiciliar; (b) fatores intra-individuais: hábitos de vida (consumo atual de cigarros e ter consumido cinco ou mais drinques de qualquer tipo de bebida alcoólica quase todos os dias, em algum período da vida) e indicadores da condição de saúde (história médica de artrite e de doença coronariana, hipertensão arterial [estágio 1: pressão arterial sistólica $=140-159 \mathrm{mmHg} \mathrm{e} / \mathrm{ou}$ pressão diastólica $=90-99 \mathrm{mmHg}$; estágio 2: pressão arterial sistólica Ž $160 \mathrm{mmHg}$ ou pressão arterial diastólica $\breve{Z} 100 \mathrm{mmHg}$, índice de massa corporal [IMC], níveis séricos de colesterol, glicose em jejum e sorologia para o T. cruzi); (c) fatores extra-individuais: suporte social (número de visitas regulares dos filhos no último mês, número de visitas regulares de parentes no último mês, número de amigos que encontra pelo menos uma vez por mês) e indicadores da utilização de serviços de saúde (número de visitas ao médico e de internações hospitalares nos últimos 12 meses; filiação a plano privado de saúde; número de medicamentos prescritos usados nos últimos três meses).

Além dessas variáveis, considerou-se a presença de um cuidador em caso de doença, definida pela pergunta: "Quando adoece, quem cui- da do(a) senhor(a)?". Maiores detalhes podem ser vistos em Lima-Costa et al. 16.

\section{Fontes de informação}

As informações foram obtidas por meio de entrevistas, medidas físicas e exames laboratoriais. As entrevistas foram realizadas no domicílio dos participantes, utilizando-se o ques tionário The Bambuí Health and Aging Study (BHAS). Quando o entrevistado estava impossibilitado de responder a entrevista devido a algum problema de saúde ou a um prejuízo cognitivo, um respondente próximo foi utilizado; este, entretanto, não respondeu perguntas que requeriam julgamento do entrevistado. As medidas físicas de todos os participantes foram tomadas no Posto Emmanuel Dias ou no domicílio, quando havia uma condição de saúde limitante. Para a medida da pressão arterial foram utilizados esfigmomanômetros de mercúrio de mesa padrão e estetoscópios. Peso, altura, circunferências do braço, do pulso, do quadril e prega cutânea do tríceps foram medidos utilizando equipamentos padronizados. As dosagens de glicose e colesterol total foram realizadas em jejum utilizando um analisador automatizado. Sorologia para o T. cruzi foi baseada nos testes de hemaglutinação e ELISA. Maiores informações podem ser obtidas em Lima-Costa et al. 16 .

\section{Análise dos dados}

As características dos idosos que necessitavam de cuidador foram comparadas àquelas dos idosos que não necessitavam. A análise dos dados foi baseada no teste do qui-quadrado de Pearson, qui-quadrado para tendência linear (análise bivariada) e estimativas de odds ratio e dos respectivos intervalos de confiança em nível de 95\% (método de Woolf).

A análise multivariada foi realizada utilizando-se o método de regressão logística múltipla 21 . As variáveis que apresentaram associação com a variável dependente em nível de significância inferior a 0,20 na análise bivariada foram incluídas na análise multivariada 22 . As variáveis que persistiram associadas com a necessidade de cuidador em nível de significância inferior a 0,05 foram mantidas no modelo final. Exceção a essa regra foi feita para sexo e idade porque foram consideradas a priori variáveis de confusão neste estudo e foram incluídas em todos os modelos logísticos. O programa Stata foi utilizado para as análises dos dados. 


\section{Resultados}

Dos 1.742 residentes de Bambuí com sessenta anos ou mais, 1.606 (92\%) participaram do presente trabalho (642 homens e 964 mulheres). A média de idade foi de 69,3 anos (desvio-padrão de 7,4 anos), com variação de 60 a 95 anos. Dos 1.606 idosos, 370 (23\%) necessitavam de cuidador, segundo os critérios assumidos neste trabalho, sendo 146 homens e 224 mulheres.

A distribuição das características sócio-demográficas predisponentes significantemente associadas à necessidade de cuidador está apresentada na Tabela 1. Verificou-se associação significante entre a necessidade de cuidador e faixa etária, estado conjugal, anos completos de escolaridade, renda domiciliar e o tipo de arranjo domiciliar. Sexo $(\mathrm{p}=0,817)$ e procedência ( $\mathrm{p}=0,843$ ) não apresentaram associação significativa com necessidade de cuidador.

Os fatores intra-individuais (hábitos de vida e indicadores da condição de saúde) que apresentaram associação estatisticamente significante estão demonstrados na Tabela 2. O relato de ter consumido cinco ou mais drinques de qualquer tipo de bebida alcoólica, quase todos os dias, em algum período da vida, esteve associado à necessidade de cuidador, enquanto o consumo atual e passado de cigarros $(\mathrm{p}=$ 0,621 ) não apresentou tal associação. História médica de artrite, de coronariopatia, de hipertensão arterial, IMC, níveis séricos de colesterol e sorologia para T. cruzi apresentaram associações significantes com a necessidade de cuidador. Glicemia em jejum ( $p=0,750)$ não apresentou associação significante com a necessidade de cuidador.

Na Tabela 3 está apresentada a distribuição dos fatores extra-individuais (indicadores de suporte social e de utilização de serviços de saúde) significantemente associados à necessidade de cuidador entre idosos. Associações significantes foram encontradas com o número de visitas dos filhos no último mês. As visitas de outros parentes e o encontro com amigos não mostraram associação significante com a necessidade de cuidador ( $p=0,360$ e $p=0,129$, respectivamente). Verificaram-se associações positivas com o número de visitas ao médico nos últimos 12 meses, o número de medicamentos prescritos e usados nos últimos noventa dias e o número de internações hospitalares nos últimos 12 meses. Associação negativa foi observada para a filiação a plano privado de saúde.

Os resultados finais da análise multivariada dos fatores associados à necessidade de cuidador entre idosos encontram-se na Tabela 4. Os fatores que apresentaram associações independentes e positivas com a necessidade de cuidador entre idosos após ajustamento por variáveis de confusão foram: (a) fatores predisponentes: associações positivas e independentes foram demonstradas para a faixa etária (70-79 anos; 80 anos e mais) e o estado conjugal (ser solteiro). Associações independentes e negativas foram encontradas para número de anos completos de escolaridade (1-3; 4-7 e Ž 8), renda domiciliar em salários-mínimos da época (2-3,9 e Ž 4) e tipo de arranjo domiciliar (mora sozinho); (b) fatores intra-individuais: estiveram associados positiva e independentemente com a necessidade de cuidador: o consumo regular de cinco ou mais drinques de bebida alcoólica em algum período da vida, história médica de hipertensão arterial (estágio 2) e IMC superior a $29 \mathrm{~kg} / \mathrm{m}^{2}$. A dosagem sérica de colesterol (> 240mg\%) esteve negativa e independentemente associada à necessidade de cuidador; (c) fatores extra-individuais: associação positiva e independente foi notada para o número de medicamentos prescritos (2-3 e Ž 4) utilizados nos últimos noventa dias, enquanto a filiação a plano privado de saúde mostrou associação negativa.

Na Tabela 5 encontra-se a distribuição do cuidador apontado pelo idoso quando este adoece. Filha, outro familiar e outra pessoa que não membro da família foram com mais freqüência apontados como cuidadores entre idosos incapacitados do que entre os demais.

\section{Discussão}

Os resultados deste trabalho mostram que, na comunidade estudada, uma parcela considerável da população idosa (23\%) apresenta necessidade de cuidador. Esta proporção é razoavelmente semelhante à de incapacidades estimadas para a população idosa americana nas décadas de 80 e 9023

O envelhecimento é um fenômeno diferencial para homens e mulheres. As mulheres são mais longevas que os homens e tendem a apresentar mais incapacidades do que os mesmos 14. No entanto, no presente trabalho, homens e mulheres apresentaram proporções semelhantes de necessidade de cuidador, independentemente da faixa etária (dados não apresentados). Maiores investigações são necessárias para compreender tal fenômeno.

Entre os idosos bambuienses, a necessidade de cuidador aumentou com a idade, o que é consistentemente observado em diferentes populações 11. Guralnik et al. 24 sugerem que a ca- 
Distribuição das características sócio-demográficas predisponentes significantemente associadas

à necessidade de cuidador entre idosos. Projeto Bambuí, Minas Gerais, Brasil, 1997.

\begin{tabular}{|c|c|c|c|}
\hline \multirow{2}{*}{$\begin{array}{l}\text { Variável (características } \\
\text { sócio-demográficas) }\end{array}$} & \multicolumn{2}{|c|}{ Necessidade de cuidador } & \multirow[t]{2}{*}{ OR (IC95\%) } \\
\hline & $\begin{array}{c}\operatorname{Sim}(n=370) \\
\%\end{array}$ & $\begin{array}{c}\text { Não }(n=1.236) \\
\%\end{array}$ & \\
\hline \multicolumn{4}{|l|}{ Sexo } \\
\hline Masculino & 39,5 & 40,1 & 1,00 \\
\hline \multirow[t]{2}{*}{ Feminino } & 60,5 & 59,9 & $1,03(0,81-1,30)$ \\
\hline & $p=0,817$ & & \\
\hline \multicolumn{4}{|l|}{ Faixa etária (em anos) } \\
\hline $60-69$ & 41,6 & 63,0 & 1,00 \\
\hline $70-79$ & 33,5 & 29,7 & $1,71(1,31-2,23)$ \\
\hline \multirow[t]{2}{*}{$\geq 80$} & 24,9 & 7,3 & $5,17(3,69-7,25)$ \\
\hline & $p^{\prime}=0,000$ & & \\
\hline \multicolumn{4}{|l|}{ Estado conjugal } \\
\hline Casado/união consensual & 41,6 & 51,1 & 1,00 \\
\hline Solteiro & 15,4 & 9,1 & $2,07(1,44-2,97)$ \\
\hline Divorciado/separado & 4,1 & 5,4 & $0,92(0,51-1,65)$ \\
\hline \multirow[t]{2}{*}{ Viúvo } & 38,9 & 34,4 & $1,39(1,07-1,80)$ \\
\hline & $p=0,000$ & & \\
\hline \multicolumn{4}{|c|}{ Anos completos de escolaridade } \\
\hline Nenhum & 49,2 & 27,7 & 1,00 \\
\hline $1-3$ & 29,4 & 33,7 & $0,49(0,37-0,65)$ \\
\hline $4-7$ & 17,4 & 29,6 & $0,33(0,24-0,46)$ \\
\hline$\geq 8$ & 4,0 & 9,0 & $0,26(0,14-0,45)$ \\
\hline \multirow[t]{2}{*}{ Sem informação } & 0,01 & - & - \\
\hline & $p^{\prime}=0,000$ & & \\
\hline \multicolumn{4}{|c|}{$\begin{array}{l}\text { Renda domiciliar mensal } \\
\text { (em salários mínimos da época) }\end{array}$} \\
\hline$<1$ & 36,8 & 27,7 & 1,00 \\
\hline $1,0-1,9$ & 40,3 & 36,6 & $0,83(0,63-1,09)$ \\
\hline $2,0-3,9$ & 9,7 & 16,2 & $0,45(0,30-0,68)$ \\
\hline$\geq 4$ & 11,3 & 18,8 & $0,46(0,31-0,67)$ \\
\hline \multirow[t]{2}{*}{ Sem informação } & 1,9 & 0,7 & - \\
\hline & $p^{\prime}=0,000$ & & \\
\hline \multicolumn{4}{|c|}{$\begin{array}{l}\text { Tipo de arranjo domiciliar } \\
\text { (número de gerações no domicílio) }\end{array}$} \\
\hline 1 & 17,8 & 22,4 & 1,00 \\
\hline Vive só & 11,6 & 17,0 & $0,86(0,56-1,31)$ \\
\hline 2 & 47,3 & 40,8 & $1,46(1,06-2,00)$ \\
\hline 3 & 20,3 & 19,7 & $1,30(0,89-1,88)$ \\
\hline \multirow[t]{2}{*}{ Sem informação } & 3,0 & 0,1 & - \\
\hline & $p^{\prime}=0,000$ & & \\
\hline
\end{tabular}

$p=$ teste qui-quadrado de Pearson; $p^{\prime}=$ teste do qui-quadrado para tendência linear.

da aumento de dez anos em idade haja o dobro de risco de declínio do status funcional. Na população estudada a incapacidade funcional aumentou 3,1 vezes na faixa de 80 anos e $1,5 \mathrm{vez}$ na de 70 a 79 anos, quando comparada à faixa etária de 60 a 69 anos.

$\mathrm{O}$ isolamento social é fator de risco para incapacidade 25,26 . Sabe-se que a direção das cor- relações entre estado marital e status funcional depende do gênero, do ambiente social e das condições sócio-econômicas 11. Em países desenvolvidos, a dependência está associada à viuvez sem filhos 26. Em Bambuí, o fato de ser solteiro esteve significantemente associado à necessidade de cuidador. Pode-se especular se esta diferença não é devida ao fato de que $93 \%$ 
Características intra-individuais (hábitos de vida e indicadores das condições de saúde) significantemente associadas à necessidade de cuidador entre idosos. Projeto Bambuí, Minas Gerais, Brasil, 1997.

\begin{tabular}{|c|c|c|c|}
\hline \multirow[t]{2}{*}{ Características intra-individuais } & \multicolumn{2}{|c|}{ Necessidade de cuidador } & \multirow[t]{2}{*}{ OR (IC95\%) } \\
\hline & $\begin{array}{c}\operatorname{Sim}(n=370) \\
\%\end{array}$ & $\begin{array}{c}\text { Não }(n=1.236) \\
\%\end{array}$ & \\
\hline \multicolumn{4}{|l|}{ Hábitos de vida } \\
\hline \multicolumn{4}{|c|}{$\begin{array}{l}\text { Consumo de pelo menos cinco drinques diários } \\
\text { de bebida alcoólica em algum período da vida }\end{array}$} \\
\hline Não & 82,7 & 88,7 & 1,00 \\
\hline Sim & 16,8 & 11,2 & $1,60(1,15-2,21)$ \\
\hline \multirow[t]{2}{*}{ Sem informação } & 0,5 & 0,1 & - \\
\hline & $p=0,000$ & & \\
\hline \multicolumn{4}{|l|}{ Indicadores da condição de saúde } \\
\hline \multicolumn{4}{|c|}{ História de diagnóstico médico de artrite } \\
\hline Não & 75,8 & 70,0 & 1,00 \\
\hline \multirow[t]{2}{*}{ Sim } & 24,2 & 30,0 & $1,34(1,04-1,74)$ \\
\hline & $p=0,025$ & & \\
\hline \multicolumn{4}{|l|}{$\begin{array}{l}\text { História de diagnóstico médico } \\
\text { de doença coronariana }\end{array}$} \\
\hline Não & 84,1 & 89,4 & 1,00 \\
\hline Sim & 14,6 & 9,6 & $1,61(1,14-2,28)$ \\
\hline \multirow[t]{2}{*}{ Sem informação } & 1,3 & 1,0 & - \\
\hline & $p=0,006$ & & \\
\hline \multicolumn{4}{|l|}{ Hipertensão arterial* } \\
\hline Não & 46,0 & 52,8 & 1,00 \\
\hline Estágio 1 & 20,2 & 25,2 & $0,93(0,68-1,25)$ \\
\hline Estágio 2 & 21,6 & 16,6 & $1,50(1,10-2,04)$ \\
\hline \multirow[t]{2}{*}{ Sem informação } & 12,2 & 5,4 & - \\
\hline & $p^{\prime}=0,014$ & & \\
\hline \multicolumn{4}{|l|}{ Índice de massa corporal** $\left(\mathrm{kg} / \mathrm{m}^{2}\right)$} \\
\hline $25-29$ & 31,2 & 34,4 & 1,00 \\
\hline$<25$ & 40,3 & 48,5 & $1,19(0,89-1,59)$ \\
\hline$>29$ & 12,7 & 11,5 & $1,58(1,06-2,36)$ \\
\hline \multirow[t]{2}{*}{ Sem informação } & 22,9 & 5,6 & - \\
\hline & $p^{\prime}=0,000$ & & \\
\hline \multicolumn{4}{|l|}{ Colesterol total (mg\%) } \\
\hline$<200$ & 26,5 & 22,2 & 1,00 \\
\hline $200-239,9$ & 31,1 & 32,4 & $0,81(0,59-1,10)$ \\
\hline$\geq 240$ & 30,5 & 40,0 & $0,64(0,47-0,87)$ \\
\hline \multirow[t]{2}{*}{ Sem informação } & 11,9 & 5,4 & - \\
\hline & $p^{\prime}=0,018$ & & \\
\hline \multicolumn{4}{|c|}{ Soropositividade para o Trypanosoma cruzi } \\
\hline Não & 46,5 & 61,1 & 1,00 \\
\hline Sim & 41,6 & 33,6 & $1,63(1,27-2,09)$ \\
\hline \multirow[t]{2}{*}{ Sem informação } & 11,9 & 5,3 & - \\
\hline & $p=0,000$ & & \\
\hline
\end{tabular}

$\mathrm{p}=$ teste qui-quadrado de Pearson; $\mathrm{p}^{\prime}=$ valor de $\mathrm{p}$ (teste do qui-quadrado para tendências lineares);

* Hipertensão arterial $=$ não: pressão arterial sistólica $\leq 139 \mathrm{mmHg}$ e pressão diastólica $<90 \mathrm{mmHg}$

(Estágio 1 = pressão arterial sistólica $=140-159 \mathrm{mmHg}$ e/ou pressão diastólica $=90-99 \mathrm{mmHg}$,

Estágio 2 = pressão arterial sistólica $\geq 160 \mathrm{mmHg}$ ou pressão arterial diastólica $\geq 100 \mathrm{mmHg}$ );

** Índice de massa corporal = peso/altura. 
Características extra-individuais (indicadores de suporte social e de utilização de serviços de saúde) significantemente associados à necessidade de cuidador, entre idosos da comunidade. Projeto Bambuí, Minas Gerais, Brasil, 1997.

\begin{tabular}{|c|c|c|c|}
\hline \multirow[t]{2}{*}{ Características } & \multicolumn{2}{|c|}{ Necessidade de cuidador } & \multirow[t]{2}{*}{ OR (IC95\%) } \\
\hline & $\begin{array}{c}\operatorname{Sim}(n=370) \\
\%\end{array}$ & $\begin{array}{c}\text { Não }(n=1.236) \\
\%\end{array}$ & \\
\hline \multicolumn{4}{|l|}{ Indicadores de suporte social } \\
\hline \multicolumn{4}{|l|}{$\begin{array}{l}\text { Número de visitas regulares dos filhos } \\
\text { no último mês }\end{array}$} \\
\hline Diariamente ou quase/mora junto & 39,8 & 38,4 & 1,00 \\
\hline $1-4$ vezes/semana & 17,0 & 18,9 & $0,88(0,63-1,23)$ \\
\hline $1-3$ vezes/mês & 14,1 & 19,2 & $0,71(0,50-1,01)$ \\
\hline$<1 \mathrm{vez} / \mathrm{mês}$ & 7,6 & 10,9 & $0,68(0,43-1,06)$ \\
\hline Não tem filhos & 21,4 & 12,7 & $1,63(1,18-2,27)$ \\
\hline \multirow[t]{2}{*}{ Sem informação } & 0,5 & 0,1 & - \\
\hline & $p=0,000$ & & \\
\hline \multicolumn{4}{|c|}{ Indicadores de utilização de serviços de saúde } \\
\hline \multicolumn{4}{|c|}{$\begin{array}{l}\text { Número de visitas a um médico } \\
\text { nos últimos } 12 \text { meses }\end{array}$} \\
\hline 0 & 14,3 & 21,0 & 1,00 \\
\hline 1 & 17,3 & 21,7 & $1,17(0,78-1,74)$ \\
\hline 2 & 15,7 & 14,9 & $1,54(1,01-2,34)$ \\
\hline 3 & 14,0 & 12,4 & $1,65(1,07-2,54)$ \\
\hline \multirow[t]{2}{*}{$\geq 4$} & 38,7 & 30,0 & $1,88(1,32-2,68)$ \\
\hline & $p^{\prime}=0,003$ & & \\
\hline \multicolumn{4}{|c|}{ Número de hospitalizações nos últimos 12 meses } \\
\hline 0 & 67,0 & 80,6 & 1,0 \\
\hline 1 & 20,5 & 14,1 & $1,75(1,30-2,38)$ \\
\hline \multirow[t]{2}{*}{$\geq 2$} & 12,5 & 5,3 & $2,80(1,87-4,18)$ \\
\hline & $p=0,000$ & & \\
\hline \multicolumn{4}{|l|}{ Filiação a plano privado de saúde } \\
\hline Não & 89,2 & 78,2 & 1,0 \\
\hline \multirow[t]{2}{*}{ Sim } & 10,8 & 21,8 & $0,43(0,30-0,62)$ \\
\hline & $p=0,000$ & & \\
\hline \multicolumn{4}{|l|}{$\begin{array}{l}\text { Número de medicamentos prescritos } \\
\text { e usados nos últimos } 90 \text { dias }\end{array}$} \\
\hline 0 & 11,4 & 22,9 & 1,00 \\
\hline 1 & 11,9 & 14,9 & $1,61(1,02-2,56)$ \\
\hline $2-3$ & 28,9 & 29,6 & $1,97(1,33-2,90)$ \\
\hline \multirow[t]{2}{*}{$\geq 4$} & 47,8 & 32,6 & $2,96(2,05-4,28)$ \\
\hline & $p^{\prime}=0,000$ & & \\
\hline
\end{tabular}

$p=$ teste qui-quadrado de Pearson; $p^{\prime}=$ teste do qui-quadrado de tendência linear.

dos idosos viúvos de Bambuí possuíam filhos, enquanto que $68 \%$ das idosas solteiras e $32 \%$ dos solteiros não os tinham (dados não mostrados).

Maior nível educacional está associado com melhor status funcional 11 e menor risco para incapacidade cognitiva 27 . Os resultados do presente trabalho são consistentes com essa observação: a escolaridade foi um fator forte, negativa e independentemente associado à necessidade de cuidador.
Da mesma forma, a renda domiciliar mostrou associação negativa e independente com a necessidade de cuidador entre os idosos bambuienses. É na parcela mais pobre que se encontra a maioria dos idosos incapacitados (79\% dos que necessitavam de cuidador tinham renda familiar menor do que dois salários-mínimos da época). Segundo Camarano et al. 28 , educação e rendimentos estão fortemente associados a melhores condições de saúde. Dessa forma, a necessidade de cuidador tam- 
bém reflete a desigualdade da distribuição de renda e da oportunidade de estudo entre os idosos de Bambuí.

Quanto aos arranjos domiciliares, 15\% dos idosos bambuienses moravam sozinhos. No presente estudo, verifica-se que a principal fonte de suporte para a população de idosos ainda é a família que coabita com eles em domicílios multigeracionais. Os arranjos multigeracionais, mais do que uma opção sociocultural, mostraram-se uma forma de sobrevivência dos idosos brasileiros 29. Os idosos residentes nesses domicílios representam uma parcela da população idosa que tende a ser mais pobre, com mais problemas de saúde e mais dependência no dia-adia do que a média de idosos. Concordando com tais observações, em Bambuí o fato de morar sozinho esteve associado negativa e independentemente com a necessidade de cuidador. Isso pode refletir melhores condições daqueles que são capazes de morar sozinhos comparativamente aos que coabitam com mais gerações.

Dentre os fatores intra-individuais investigados no presente estudo, o consumo de, pelo menos, cinco drinques diários de bebida alcoólica em algum período da vida mostrou associação positiva e independente com a necessidade de cuidador entre idosos. Essa associação deve estar subestimada em razão do viés de sobrevivência, porque existem evidências de que o consumo pesado de álcool é fator de risco para mortalidade precoce 30 . Ressalte-se que o consumo de álcool tem sido pouco estudado na população idosa 31 .

A história de hipertensão arterial esteve positiva e independentemente associada com a necessidade de cuidador. A relação entre pressão arterial e risco de doença cardiovascular é contínua, consistente e independente de outros fatores de risco 32 . Quanto maior a pressão arterial, maior o risco de infarto do miocárdio, insuficiência cardíaca, acidente vascular cerebral e doença renal. As doenças do aparelho circulatório são a maior causa de mortalidade em idosos brasileiros, aumentando com a idade 33. A hipertensão arterial é relevante do ponto de vista de saúde pública não somente pela alta prevalência entre idosos, mas principalmente pelas complicações relacionadas como as doenças cerebrovasculares, incluindo a demência vascular, e pela facilidade do seu tratamento uma vez diagnosticada. Apesar disso, Firmo et al. 34 demonstraram em outro estudo que, em Bambuí, o controle adequado da pressão arterial é inferior ao recomendado pela OMS (37\% dos idosos hipertensos não estavam sendo tratados para essa condição e $61 \%$ dos idosos bambuienses apesar do tratamento an-
Tabela 4

Resultados da análise multivariada das características sócio-demográficas predisponentes e dos fatores intra e extra-individuais, entre os idosos da comunidade que necessitam de cuidador. Projeto Bambuí, Minas Gerais, Brasil, 1997.

\begin{tabular}{|c|c|}
\hline Variáveis & OR ajustado (IC95\%) \\
\hline \multicolumn{2}{|c|}{ Características sócio-demográficas predisponentes } \\
\hline \multicolumn{2}{|c|}{ Faixa etária (em anos) } \\
\hline $60-69$ & 1,00 \\
\hline $70-79$ & $1,77(1,28-2,43)$ \\
\hline$\geq 80$ & $4,35(2,77-6,83)$ \\
\hline \multicolumn{2}{|l|}{ Estado conjugal } \\
\hline Casado & 1,00 \\
\hline Solteiro & $2,28(1,34-3,87)$ \\
\hline Divorciado/separado & $0,94(0,45-1,99)$ \\
\hline Viúvo & $1,02(0,67-1,56)$ \\
\hline \multicolumn{2}{|c|}{ Anos completos de escolaridade } \\
\hline 0 & 1,00 \\
\hline $1-3$ & $0,55(0,39-0,77)$ \\
\hline $4-7$ & $0,43(0,29-0,64)$ \\
\hline 8 & $0,34(0,15-0,63)$ \\
\hline \multicolumn{2}{|c|}{ Renda domiciliar (em salários-mínimos da época) } \\
\hline$<1$ & 1,00 \\
\hline $1,0-1,9$ & $0,79(0,55-1,14)$ \\
\hline $2,0-3,9$ & $0,49(0,29-0,82)$ \\
\hline$\geq 4$ & $0,58(0,34-0,99)$ \\
\hline \multicolumn{2}{|c|}{$\begin{array}{l}\text { Tipo de arranjo domiciliar (número } \\
\text { de gerações no domicílio) }\end{array}$} \\
\hline 1 & 1,00 \\
\hline Vive só & $0,43(0,23-0,78)$ \\
\hline 2 & $1,29(0,83-2,01)$ \\
\hline 3 & $1,28(0,78-2,11)$ \\
\hline \multicolumn{2}{|c|}{ Características intra-individuais } \\
\hline \multicolumn{2}{|c|}{$\begin{array}{l}\text { Ter consumido cinco ou mais drinques de bebida } \\
\text { alcoólica, quase todos os dias, em algum período da vida }\end{array}$} \\
\hline Não & 1,00 \\
\hline Sim & $2,39(1,55-3,69)$ \\
\hline \multicolumn{2}{|l|}{ Hipertensão arterial* } \\
\hline Não & 1,00 \\
\hline Estágio 1 & $1,03(0,73-1,47)$ \\
\hline Estágio 2 & $1,55(1,08-2,20)$ \\
\hline \multicolumn{2}{|c|}{ Índice de massa corporal ${ }^{\star \star}\left(\mathrm{kg} / \mathrm{m}^{2}\right)$} \\
\hline $25-29$ & 1,00 \\
\hline$<25$ & $0,88(0,63-1,22)$ \\
\hline$>30$ & $1,66(1,07-2,58)$ \\
\hline \multicolumn{2}{|l|}{ Colesterol total (mg\%) } \\
\hline$<200$ & 1,00 \\
\hline $200-239,9$ & $0,73(0,51-1,05)$ \\
\hline$>240$ & $0,59(0,41-0,85)$ \\
\hline \multicolumn{2}{|c|}{ Características extra-individuais } \\
\hline \multicolumn{2}{|c|}{$\begin{array}{l}\text { Número de medicamentos prescritos usados } \\
\text { nos últimos } 90 \text { dias }\end{array}$} \\
\hline 0 & 1,00 \\
\hline 1 & $1,29(0,74-2,24)$ \\
\hline $2-3$ & $1,75(1,10-2,76)$ \\
\hline$\geq 4$ & $2,79(1,77-4,37)$ \\
\hline \multicolumn{2}{|c|}{ Filiação a plano privado de saúde } \\
\hline Não & 1,00 \\
\hline Sim & $0,63(0,41-0,97)$ \\
\hline
\end{tabular}

OR ajustado = odds ratio ajustado pelas variáveis listadas na tabela e sexo, utilizando regressão logística multivariada. O modelo logístico final incluiu 1.432 idosos; * hipertensão arterial = não: pressão arterial sistólica $\leq 139 \mathrm{mmHg}$ e pressão diastólica $<90 \mathrm{mmHg}$ (Estágio $1=$ pressão arterial sistólica $=140-159 \mathrm{mmHg}$ e/ou pressão diastólica $=90-99 \mathrm{mmHg}$, Estágio $2=$ pressão arterial sistólica $\geq 160 \mathrm{mmHg}$ ou pressão diastólica $\geq 100 \mathrm{mmHg}$; ${ }^{\star *}$ Índice de massa corporal = peso/altura. 
Tabela 5

Distribuição do tipo de cuidador (grau de parentesco com o idoso)

em caso de doença, entre idosos que apresentam necessidade de cuidador.

Projeto Bambuí, Minas Gerais, Brasil, 1997.

\begin{tabular}{|c|c|c|c|}
\hline \multirow{2}{*}{$\begin{array}{l}\text { Quem é o cuidador } \\
\text { em caso de doença }\end{array}$} & \multicolumn{2}{|c|}{ Necessidade de cuidador } & \multirow[t]{2}{*}{ OR (IC95\%) } \\
\hline & $\begin{array}{c}\operatorname{Sim}(n=370) \\
\%\end{array}$ & $\begin{array}{c}\text { Não }(n=1.236) \\
\%\end{array}$ & \\
\hline Ninguém & 3,0 & 6,5 & 1,00 \\
\hline Cônjuge & 27,8 & 37,2 & $1,62(0,84-3,16)$ \\
\hline Filha & 36,0 & 34,5 & $2,27(1,17-4,39)$ \\
\hline Outro familiar & 24,0 & 18,3 & $2,86(1,46-5,63)$ \\
\hline Outra pessoa & 8,4 & 3,5 & $5,24(2,40-11,5)$ \\
\hline \multirow[t]{2}{*}{ Sem informação } & 0,8 & 0,08 & - \\
\hline & $p=0,000$ & & \\
\hline
\end{tabular}

$\mathrm{p}=$ teste do qui-quadrado de Pearson. sitaram de cuidador consumiram maior número de medicamentos prescritos, refletindo suas piores condições de saúde 39 . Por outro lado cabe indagar se essa associação não ocorre na direção oposta. Nesse caso, a iatrogenia estaria envolvida nessa associação. A polimedicação é freqüentemente acompanhada de polipatologia, outro fator de risco para a iatrogenia 40 . Estima-se que cerca de um terço das prescrições medicamentosas para idosos no domicílio são duvidosas ou mesmo, inúteis 41 . A iatrogenia também favorece a incapacidade funcional e deve ser entendida como um problema de saúde pública 42,43 . São necessárias investigações para verificar a contribuição da adequação da prescrição medicamentosa na população estudada.

A filiação a plano privado de saúde também mostrou associação independente e negativa com a necessidade de cuidador. Entre os idosos que dependem exclusivamente dos serviços públicos de saúde encontram-se os que necessitam de cuidador. Assim, a incapacidade é ônus para o Estado.

Em Bambuí, verifica-se que as principais fontes de cuidados para os idosos, em caso de doença, são o cônjuge ou a filha, seguido por outro familiar. Entretanto, quando aqueles que necessitam de cuidador são comparados aos que não necessitam, observa-se que a associação mais forte é para uma pessoa externa à família, seguida por outro familiar e pela filha. Esses resultados podem estar refletindo a mudança dos arranjos familiares apontada por Camarano 44 , com a nuclearização das famílias e o trabalho das mulheres fora de casa, modificando a figura de cuidador tradicional (esposa responsável pelas tarefas domésticas, inclusive o cuidado de crianças, idosos e enfermos, ou filha solteira que não teve ocupação prévia).

Utilizando os critérios sugeridos por Verbrugge \& Jette 7, encontramos associação estatisticamente significante entre a necessidade de cuidador e os seguintes fatores: (a) predisponentes: faixa etária (70-79 anos, 80 anos e mais), estado conjugal (ser solteiro), número de anos completos de escolaridade (1-3, 4-7 e Ž 8), renda domiciliar em salários-mínimos da época (2-3, 9 e Ž 4) e tipo de arranjo domiciliar (mora sozinho); (b) intra-individuais: o consumo regular de cinco ou mais drinques de bebida alcoólica em algum período da vida, história médica de hipertensão arterial (estágio 2), IMC superior a $29 \mathrm{~kg} / \mathrm{m}^{2}$ e dosagem sérica de colesterol (> 240mg\%); (c) extra-individuais: o número de medicamentos prescritos (2-3 e Ž 4) utilizados nos últimos noventa dias e a filiação a plano privado de saúde. 
O banco de dados utilizado para o presente trabalho apresenta inúmeras vantagens: (1) estudo de base populacional (Projeto Bambuí), com idosos da comunidade e, portanto, não sujeito às tendenciosidades de estudos conduzidos em população idosa institucionalizada; (2) validade interna e controle de qualidade da informação, assegurados a partir da grande aderência e participação da população 16. Além disso, a existência de um grande conjunto de informações possibilitou a investigação de variáveis predisponentes, intra-individuais e extra-individuais e seu ajustamento por variáveis de confusão. Finalmente, a principal limitação metodológica deste trabalho é o seu delineamento seccional, impossibilitando qualquer inferência causal.

\section{Resumo}

Este estudo tem por objetivo determinar a prevalência e os fatores associados com a necessidade de cuidador entre idosos residentes na comunidade. Foram selecionados todos os residentes na cidade de Bambuí, Minas Gerais, Brasil, com $\geq 60$ anos de idade $(n=1.742)$. Destes, $92 \%$ foram entrevistados $e$ 86\% examinados. A variável dependente - necessidade de cuidador - foi definida como: (1) relato de incapacidade para realizar pelo menos uma das atividades da vida diária elou (2) escore inferior a 13 no Mini Mental State Examen. A prevalência da necessidade de cuidador foi de $23 \%$. Apresentaram associações positivas e independentes com a necessidade de cuidador: idade, ser solteiro, história de alcoolismo prévio, hipertensão arterial, obesidade e uso de medicamentos prescritos. Observaram-se associações negativas e independentes para: escolaridade, renda familiar, viver só, colesterol total $>240 \mathrm{~mm} H g$ e ter plano privado de saúde. Assim, a necessidade de cuidador em Bambuí estava associada a piores condições sócio-econômicas e de saúde. O cuidado dos idosos dependentes é um problema de saúde pública.

Idoso Débil; Cuidadores; Saúde do Idoso; Condições de Saúde
Os resultados do presente trabalho mostram uma alta prevalência da necessidade de cuidador em uma população idosa que não dispõe de recursos assistenciais comunitários comparáveis aos dos países desenvolvidos. A família ainda é a principal instituição que oferece esse suporte e os arranjos multigeracionais são, antes, uma questão de sobrevivência do que circunstanciais. Chama a atenção, entre os nossos resultados, a associação entre a necessidade de cuidador e a baixa escolaridade e a renda domiciliar. Os idosos mais carentes devido à baixa escolaridade, baixa renda pessoal e/ou domiciliar são os que necessitam de cuidador. Esse fato precisa ser considerado ao se programar políticas de saúde para idosos.

\section{Colaboradores}

K. C. Giacomin participou do delineamento do estudo, realizou a análise dos dados e foi a redatora principal do artigo. M. F. Lima-Costa é a coordenadora geral do projeto de pesquisa, tendo orientado o delineamento do estudo, participado da análise e discussão dos resultados e da redação final do trabalho. E. Uchôa participou da discussão do delineamento do estudo, da discussão dos resultados e revisão crítica final do artigo. J. O. A. Firmo foi coordenadora do trabalho de campo, tendo colaborado na análise dos dados e revisão crítica final do artigo. 


\section{Referências}

1. Lima-Costa MF, Veras R. Saúde pública e envelhecimento. Cad Saúde Pública 2003; 19:700-1.

2. Fried LP, Guralnik JM. Disability in older adults: evidence regarding significance, etiology and risk. J Am Geriatr Soc 1997; 45:92-100.

3. Caldas CP. Envelhecimento com dependência: responsabilidades e demandas da família. Cad Saúde Pública 2003; 19:773-81.

4. Instituto Brasileiro de Geografia e Estatística. Censo demográfico, 2000. Rio de Janeiro: Instituto Brasileiro de Geografia e Estatística; 2002.

5. World Health Organization. International classification of impairments, disabilities and handicaps: a manual of classification relating to the consequences of disease. Geneva: World Health Organization; 1980.

6. Hébert R. La perte d'autonomie. Neurologie, Psychiatrie, Gériatrie 2003; 3:33-40.

7. Verbrugge LM, Jette AM. The disablement process. Soc Sci Med 1994; 38:1-14.

8. Rosa TEC, Benício MHA, Latorre MRDO, Ramos LR. Fatores determinantes da capacidade funcional entre idosos. Rev Saúde Pública 2003; 37:40-8.

9. Jette AM. Disentangling the process of disablement. Soc Sci Med 1999; 48:471-2.

10. Verbrugge LM. A global disability indicator. J Aging Stud 1997; 11:337-62.

11. Stuck AE, Walthert JM, Nikolaus T, Büla CJ, Hohmann C, Beck JC. Risk factor for functional status decline in community-living elderly people: a systematic literature review. Soc Sci Med 1999; 48:445-69.

12. Gill TM, Williams CS, Richardson ED, Berkman LF, Tinetti ME. A preditive model for ADL dependence in community-living older adults based on a reduced set of cognitive status items. J Am Geriatr Soc 1997; 45:441-5.

13. Néri AL, Sommerhalder C. As várias faces do cuidador e do bem-estar do cuidador. In: Néri AL, organizador. Cuidar de idosos no contexto da família: questões psicológicas e sociais. Campinas: Editora Átomo \& Alínea; 2002. p. 9-63.

14. Grundy E. The epidemiology of aging. In: Tallis R, Fillit H, editors. Brocklehurst's textbook of geriatric medicine and gerontology. Edinburgh: Churchill Livingstone; 2003. p. 3-20.

15. Instituto Brasileiro de Geografia e Estatística. Contagem da população, 1996. Rio de Janeiro: Instituto Brasileiro de Geografia e Estatística; 1997.

16. Lima-Costa MF, Uchôa E, Guerra HL, Firmo JOA, Vidigal PG, Barreto SM. The Bambuí Health and Ageing Study (BHAS): methodological approach and preliminary results of a population-based cohort study of the elderly in Brazil. Rev Saúde Pública 2000; 34:126-35.

17. Lima-Costa MF, Barreto SM, Guerra HL, Firmo JOA, Uchôa E, Vidigal PG. Ageing with Trypanosoma cruzi infection in a community where the transmission has been interrupted: the Bambuí Health and Ageing Study (BHAS). Int J Epidemiol 2001; 30:887-93.

18. Guralnik JM, Lacroix AZ. Assessing physical function in older populations. In: Wallace RB, Woolson RF, editors. The epidemiologic study of the el- derly. New York: Oxford University Press; 1992. p 159-81.

19. Bertolucci PHF, Brucki SMD, Campacci SR, Juliano Y. O mini exame do estado mental em uma população geral: impacto da escolaridade. Arq Neuro-Psiquiatr 1994; 52:1-7.

20. Folstein MF, Folstein SE, McHugh PR. "Mini-Mental State". A practical method for grading the cognitive state of patients for the clinician. J Psychiatr Res 1975; 12:189-98.

21. Hosmer DW, Lemeshow S. Applied logistic regression. New York: John Wiley and Sons; 1989.

22. Fleiss FL. Statistical methods for rates and proportions. New York: John Wiley and Sons; 1981.

23. Kunkel SR, Applebaum RA. Estimating the prevalence of long-term disability for an aging society. J Gerontol 1992; 47:S253-60.

24. Guralnik JM, Lacroix AZ, Abbott RD, Berkman LF, Satterfield S, Evans DA, et al. Maintaining mobility in late life. I. Demographic characteristics and chronic conditions. Am J Epidemiol 1993; 137: 845-57.

25. Moritz DJ, Kasl SV, Berkman LF. Cognitive functioning and the incidence of limitations of daily living in an elderly community sample. Am J Epidemiol 1995; 141:41-9.

26. Forette B. Aspects démographiques et épidémiologiques en France. In: Arcand M, Hébert R, editors. Précis pratique de geriatrie. Québec: EDISEM; 1998. p. 15-20.

27. Gauthier S. Clinical diagnosis and management of Alzheimer's disease. 2nd Ed. London: Martin Dunitz; 2001.

28. Camarano AA, Beltrão KI, Pascom ARP, Medeiros M, Goldani AM. Como vive o idoso brasileiro? In: Camarano AA, organizador. Muito além dos 60: os novos idosos brasileiros. Rio de Janeiro: Instituto de Pesquisa Econômica Aplicada; 1999. p. 19-71.

29. Ramos LR. Epidemiologia do envelhecimento. In: Freitas EV, Py L, Nery AL, Cançado FAX, Gorzoni ML, Rocha SM, organizadores. Tratado de geriatria e gerontologia. Rio de Janeiro: Guanabara Koogan; 2002. p. 72-8.

30. LaCroix AZ, Guralnik JM, Berkman LF, Wallace $\mathrm{RB}$, Satterfield S. Maintaining mobility in late life. II. Smoking, alcohol consumption, physical activity and body mass index. Am J Epidemiol 1993; 137:858-69.

31. Hirata ES. Abuso e dependência do álcool em idosos. In: Forlenza OV, Caramelli P, organizadores. Neuropsiquiatria geriátrica. São Paulo: Atheneu; 2000. p. 641-8.

32. Chobanian AV, Bakris GL, Black HR, Cushman WC, Green LA, Izzo JL, et al. The seventh report of the joint national committee on prevention, detection, evaluation and treatment of high blood pressure: The JNC 7 Report. JAMA 2003; 289:256072 .

33. Lima-Costa MF, Guerra HL, Barreto SM, Guimarães RM. Diagnóstico da situação de saúde da população idosa brasileira: um estudo da mortalidade e das internações hospitalares públicas. Inf Epidemiol SUS 2000; 9:23-41. 
34. Firmo JOA, Barreto SM, Lima-Costa. The Bambuí Health and Aging Study (BHAS): factors associated with the treatment of hypertension in older adults in the community. Cad Saúde Pública 2003; 19:817-27.

35. Batista Filho M, Rissin A. A transição nutricional no Brasil: tendências regionais e temporais. Cad Saúde Pública 2003; 19 Suppl 1:S181-91.

36. Ministério da Saúde. Política nacional de alimentação e nutrição. Brasília: Ministério da Saúde; 2000.

37. Moriguchi HE, Michelon E, Vieira JLC. Dislipidemia em idosos. In: Freitas EV, Py L, Nery AL, Cançado FAX, Gorzoni ML, Rocha SM, organizadores. Tratado de geriatria e gerontologia. Rio de Janeiro: Guanabara Koogan; 2002. p. 239-48.

38. Kronmal RA, Cain KC, Omenn GS. Total serum cholesterol levels and mortality risk as a function of age: a report based on the Framingham data. Arch Intern Med 1993; 153:1065-73.

39. Loyola Filho AI, Uchôa E, Guerra HL, Firmo JOA, Lima-Costa MF. Prevalência e fatores associados à automedicação: resultados do Projeto Bambuí. Rev Saúde Pública 2002; 36:55-62.
40. Ramos LR. Os fármacos e o idoso. In: Gorzoni ML Toniolo Neto J, organizadores. Terapêutica clínica no idoso. São Paulo: Editora Sarvier/Associação Paulista de Medicina; 1995. p. 3-6.

41. Grenier L, Barbeau G. Pharmacologie. In: Arcand $M$, Hébert R, editors. Précis pratique de gériatrie. Québec: EDISEM; 1998. p. 701-13.

42. Sager MA, Rudberg MA. Functional decline associated with hospitalization for acute illness. Clin Geriatr Med 1998; 14:669-79.

43. Jones D, Poole C. Medicine taking by elderly: an overview. In: George CF, Woodhouse KW, Denham MJ, McLennan WJ, editors. Drug therapy in old age. Chichester: John Wiley \& Sons; 1998. p. 1-22.

44. Camarano AA. Envelhecimento da população brasileira: uma contribuição demográfica. In: Freitas EV, Py L, Nery AL, Cançado FAX, Gorzoni ML, Rocha SM, organizadores. Tratado de geriatria e gerontologia. Rio de Janeiro: Guanabara Koogan; 2002. p. 58-71.

Recebido em 15/Mar/2004

Versão final reapresentada em 14/Jul/2004

Aprovado em 19/Jul/2004 